\title{
Valorisation de quatre accessions de souchets (Cyperus esculentus L.): une bonne option de diversification de culture pour les producteurs de la localité de danay-vokgora (Mayo-danay) en zone soudano-sahélienne du Cameroun
}

\author{
Dérik Pierre SAKATAI ${ }^{*}$, Armand ABDOU BOUBA ${ }^{2}$, Jean Paul OLINA BASSALA ${ }^{3}$, \\ Jules BALNA ${ }^{1}$, Oumarou PALOU ${ }^{4}$ et Sali BOUROU ${ }^{1}$
}

${ }^{I}$ Institut de Recherche Agricole pour le Développement (IRAD), Centre Régional de Maroua, BP: 33 Maroua, Cameroun.

${ }^{2}$ Ecole Nationale Supérieure Polytechnique de Maroua, B.P./P.O. Box : 46 Maroua, Cameroun.

${ }^{3}$ Société du Développement du Coton du Cameroun (SODECOTON), BP : 302 Garoua, Cameroon.

${ }^{4}$ Institut de Recherche Agricole pour le Développement (IRAD), Centre Régional de Wakwa, BP: 65 Wakwa, Cameroun.

*Auteur correspondant ; E-mail : spierrederik@yahoo.com

\section{RÉSUMÉ}

La valorisation de certaines espèces sous-utilisées constitue des moyens palliatifs de la diversification des cultures dans certaines localités de l'Extrême-Nord. Plus spécifiquement, l'absence des connaissances sur les méthodes culturales de la culture de souchet (Cypérus esculentus L.) constitue un frein de sa production. Face à cette situation, une étude été menée dans la plaine du Mayo-Danay (Danay-vokgora) afin de transférer le paquet technologique (itinéraire technique) de production de cette culture aux producteurs en vue de substituer les cultures sensibles à la variabilité et au changement climatique. Une parcelle de démonstration (Champ Ecole Paysan (CEP)) de quatre accessions de souchet (Cyperus esculentus L.) a été mise en place. Les opérations culturales ont été mise en œuvre dans le dispositif en split plot comprenant les blocs de placettes complètement randomisées. La densité de semis et les accessions comportant quatre modalités chacun et répétées quatre fois ont été appliquées et contrôlées. L'issu des résultats révèlent qu'il n'existe pas des effets des densités de semis sur les rendements moyens en t/ha de souchet au seuil de 5\% de signification. Par contre, les accessions « $\mathrm{c}=$ glazay » et « $\mathrm{d}=$ wéchéché » se sont différenciées avec les meilleurs rendements moyens en t/ha. Bien que l'accession «c= glazay » fasse l'objet du choix de préférence des bénéficiaires de ladite localité, la comparaison des quantités nécessaires et des productivités marginales des inputs de production de souchet (Cyperus esculentus L.) ont montré qu'il serait avantageux de produire avec l'accession « $\mathrm{d}=$ wéchéché », car avec la meilleure combinaison des inputs de production, sa productivité marginale (73,09 FCFA) serait plus profitable pour l'unité additionnelle relative au coût de location de la parcelle. De ce fait, il est important d'évaluer cette pratique culturale pour mieux apprécier le degré d'adoption de l'innovation relative à l'itinéraire technique de la culture de souchet dans la localité de Danay-vokgora.

(C) 2020 International Formulae Group. All rights reserved.

Mots clés: Valorisation, Souchet, Rendement, Danay-vokgoro, Soudano-sahélienne. 


\title{
Valorization of four accessions of tigernut (Cyperus esculentus L.): a good option of cultural diversification for the producers of the locality of danay- vokgora (Mayo-danay) in sudano-sahelien zone Cameroon
}

\begin{abstract}
The development of some underutilized species is a palliative means of crop diversification in some communities in the Far North. More specifically, the lack of knowledge about the cultivation methods of Tigernut culture (Cyperus esculentus L.) is a hindrance to its production. Faced with this situation, a study was conducted in the Mayo-Danay plain (Danay-vokgora) in order to transfer the technological package (technical practice) of production of this crop to producers in order to replace crops sensitive to variability and climate change. A demonstration plot (Field School Peasant (CEP)) of four accessions of Tigernut (Cyperus esculentus L.) has been set up. To do this, the implementation of the operations was conducted in the experimental split plot including randomized completely block design. Seedling density and accessions with four modalities each and repeated four times were applied and controlled. At the end of study, the results show that at $5 \%$ level of the significance, the effects of planting densities do not influence on average yields in t/ha of Tigernut (Cyperus esculentus L.). On the other hand, "c = glazay" and "d=wéchéché" accessions of Tigernut (Cyperus esculentus L.) differed by presenting best average yields in $\mathrm{t} / \mathrm{ha}$. While "c = glazay"accession is subject to the choice of preference beneficiaries to Danay-vokgora locality (Mayo-Danay), but the comparison of quantities needed and the marginal productivity of the production of the accessions of the Tigernut (Cyperus esculentus L.) has shown that it would advantageous to produce with the "d=wéchéché" accession, because with the best combination of inputs, her marginal productivity would be more profitable for an additional unit of 73.09 FCFA for the renting cost of the parcel. Therefore, it is important to evaluate this farming practice to better appreciate the degree of adoption of innovation related to the technical practice of Tigernut (Cyperus esculentus L.) culture in the locality of Danayvokgora.
\end{abstract}

(C) 2020 International Formulae Group. All rights reserved.

Keywords: Valorization, Nutgrass, Yield, Danay-vokgoro, Sudano-sahelien.

\section{INTRODUCTION}

Le souchet (Cypérus esculentus L.) est une plante herbacée de la famille des cypéracées, originaire de la Méditerranée et de l'Est de l'Asie (Aoua et al., 2008). Il a été domestiqué et utilisé comme plante alimentaire presque dans tous les continents (Afrique, Sud de l'Europe, Asie et Nord-Est des USA et du Canada) du monde (Dodet, 2006; Defelice, 2002; De Castro et al., 2015). Cette plante est cultivée pour ses tubercules qui ont une bonne valeur nutritionnelle parce qu'elle contiennent des quantités élevées de matière grasse, de sucre, d'éléments minéraux et de vitamines (Aoua et al., 2008). Plus précisément en Afrique de l'Ouest, cette plante est connue et cultivée pour la consommation locale (OngPeamuru, 2013). Ses tubercules sont parfois moulus en farine pour diverses préparations locales, mais le plus souvent, ils sont plongés dans l'eau pour hydratation avant d'être vendus sur les marchés et dans les agences de voyages (SNV, 2006). La production de la culture du souchet contribue à plus de $95 \%$ de la production nationale du Niger, et cette culture se pratique sur l'ancien bassin arachidier de la région de Maradi (Ong-Peamuru, 2013). Cette spéculation de souchet est devenue la troisième culture d'exportation en terme de valeur pour l'entrée des devises après l'oignon et le niébé (Gambo, 2005 cité par Bori et al., 2018). La production de souchet peut contribuer à plus de 500 millions de FCFA/an en faveur des petits producteurs (Aoua et al., 2008). Le souchet est une culture de rente de haute valeur marchande au Niger car sa production serait repartie en $1 \%$ d'autoconsommation, $6 \%$ en semence et environ 93\% pour la vente (Daouda et al., 2002 cité par Bori et al., 2018; Garba, 2007). Par ailleurs, au Cameroun, la production de souchet est beaucoup plus pratiquée dans deux 
régions (Extrême-Nord et Nord). En particulier dans la région de l'Extrême Nord, le souchet est plus pratiqué dans la partie montagneuse des départements de Mayo-Sava et de MayoTsanaga. Les études sur cette spéculation n'existent pas au niveau des régions de l'Extrême-Nord et du Nord. Bori et al. (2018) ont également noté qu'au Niger, la culture du souchet prend de plus en plus d'ampleur mais les études sur ce produit sont quasiinexistantes. Pourtant, Baobab (2012) avait relevé l'importance de cette spéculation de souchet dans plusieurs domaines. Sur le plan Cosmétique, l'huile de Souchet possède des propriétés adoucissantes, hydratantes et raffermissantes pour la peau. Elle est utilisée pour la protection des cheveux, limite la repousse des poils, rend lisse et adoucit la fibre capillaire. Sur le plan alimentaire, l'huile extrait des tubercules du souchet est très stable, nutritive et savoureuse, c'est une huile idéale pour les assaisonnements. Malgré son importance capitale dans certains domaines, cette spéculation n'est pas encore bien connue par la plupart des producteurs de la partie septentrionale du Cameroun. Dans la plupart des temps, les problèmes du changement et de la variabilité climatique sont de plus en plus ressentis dans les localités de la partie septentrionale, le volume de la production de certaines spéculations baisse car leurs cycles de production n'atteignent pas la maturité physiologique. Les mêmes constats sont faits au Benin par Oloukoi et al. (2019) qui rapportent que les principales mutations pluviométriques observées par les paysannes sont le démarrage tardif, l'arrêt précoce et l'irrégularité des pluies dont les conséquences se traduisent par la baisse de la productivité et $\mathrm{du}$ rendement agricole. C'est le cas du département du Mayo-Danay dont la mauvaise répartition et les trous pluviométriques au moment de floraison, et surtout aux stades critiques des plantes ont engendré les perturbations climatiques (soient $647 \mathrm{~mm}$, $837,85 \mathrm{~mm}$ et $774,87 \mathrm{~mm}$ respectivement pour les années de 2015, 2016 et 2017(ACEFA, 2018)). Suite à cette perturbation pluviométrique, des baisses des rendements de certaines spéculations ont été enregistrées.
L'exemple la plus parfaite concerne quelques variétés des sorghos dont le rendement en $\mathrm{t} / \mathrm{ha}$ varie d'une année à une autre. En 2015, les valeurs sont de l'ordre de 1,24 t/ha; 1,13 t/ha; et 1,42 t/ha respectivement pour le sorgho de saison des pluies, sorgho de saison sèche et celle de sorgho intersaison. Par ailleurs, en 2016 pour les mêmes variétés du sorgho, ces valeurs sont de l'ordre de : 0,95 t/ha; 1,09 t/ha; 1,39 t/ha respectivement (ACEFA, 2018). Cette variabilité de rendement en baisse serait certainement causée par les perturbations climatiques alors qu'elles resteront encore prépondérantes avec d'autres spéculations cultivées de la localité. Par contre, la diversification des cultures reste des mesures palliatives pour les risques de perte en rendement des cultures. C'est pourquoi, l'intérêt porté en faveur de cette culture de souchet sous la phase d'essai dans la localité de Danay-Vokgora (Département du MayoDanay) permet aux paysans de s'approprier de la pratique culturale d'une part. D'autre part, son introduction pourrait être également un moyen de promotion du développement des activités génératrices des revenues (AGR) des petits producteurs comme la Fédération des producteurs de souchet SA'A du Maradi au Niger (CSA, 2011). En outre, l'extension de cette culture de souchet dans la zone semi-aride permet de voir la réponse de ces quatre accessions testées afin de mieux vulgariser dans d'autres localités de la région de l'Extrême-Nord en général. Autrement dit, est ce que l'introduction de la culture de souchet n'est-il pas une solution envisageable pour ce problème de variabilité climatique qui règne dans cette localité en particulier, surtout que celle-ci est moins exigeante. D'où la nécessité de la pratique du Champ Ecole Paysan (CEP) par la méthode d'un diagnostic participatif afin d'apprendre l'itinéraire technique (ITK) aux petits producteurs de cette localité de Danayvokgora (Mayo-Danay).

\section{MATÉRIEL ET MÉTHODES}

Pour la conduite de cet essai, 34 personnes volontaires et dynamiques ont été identifiés afin de bénéficier du conseil et suivi de l'itinéraire technique de la production de 
souchet. Le matériel végétal était constitué des graines de souchet (Cyperus esculents L.) issues des collectes au niveau des différents bassins (Midré, Mokolo et Mandaka) de production de département du Mayo-Tsanaga. Au total, 4 accessions ont été collectées auprès des agriculteurs de département du MayoTsanaga, dans la région de l'Extrême-Nord avant la saison des pluies. Chaque accession a été identifiée et caractérisée sur la base des ressemblances de forme, de taille et de couleur des graines, les 4 accessions ont été retenues pour l'expérimentation. L'étude a été conduite dans la localité de Danay-vokgora (Figure 1) dont la pluviométrie moyenne varie entre 647 $\mathrm{mm}$ à $837,85 \mathrm{~mm} / \mathrm{an}$ sur un sol limoneux et sableux et une température variant entre $28{ }^{\circ} \mathrm{C}$ à $40{ }^{\circ} \mathrm{C}$ (ACEFA, 2018). La planche de la photo 1 montre quatre accessions des couleurs et des tailles des grains différents constituant les matériels végétaux utilisés.

Pour la mise en place de l'essai, deux facteurs (A et B) contrôlés ont été identifiés. Le facteur variété (A) a été utilisé pour constituer quatre traitements dont les lettres correspondent aux accessions nommés en Mafa (langue locale): $\mathrm{a}=$ m'nda nkodhum; $\mathrm{b}=m^{\prime} n d a$ ged $; \mathrm{c}=$ glazay; $\mathrm{d}=$ wéchéché. Pour ce qui est du facteur densité (B), il a été constitué de quatre densités de semis dont les différentes modalités sont : $\mathrm{T}=$ témoin (semis à la volée), $\mathrm{D} 1=5 \mathrm{~cm}$ entre les lignes, $\mathrm{D} 2=10 \mathrm{~cm}$ entre les lignes et $\mathrm{D} 3=15 \mathrm{~cm}$ entre les lignes. Pour apprécier le niveau d'appropriation de l'itinéraire technique de production de souchet par les producteurs de la localité de Danayvokgora. Le choix aléatoire simple a été choisi pour constituer un échantillon de 20 bénéficiaires. A l'aide d'un questionnaire élaboré au préalable, l'interview semi structuré et individuel a été conduit pour collecter les informations auprès de chaque participant. Les données ont été codifiées dans le masque de saisi avant d'être analysé. La Figure 1 présente la localisation du site de l'essai.

\section{Dispositif expérimental}

L'aménagement manuel du champ expérimental a été réalisé le 2 juillet 2018 avec le mode de labour en monticule (Photo 2), dans un dispositif expérimental en split plot constitué des blocs de parcelles complètement randomisées ( $1 \mathrm{~m}$ entre les blocs), dont chaque facteur contrôlé comporte quatre traitements chacun et quatre répétitions. Les unités expérimentales étaient constituées en planche rectangulaire (soient $2 \mathrm{~m}$ de longueur et $1 \mathrm{~m}$ de largeur) séparées de $75 \mathrm{~cm}$ l'une de l'autre. Le nettoyage a consisté à débarrasser la parcelle de toutes qualités d'herbe. La préparation des placettes (unités expérimentales) à l'aide des houes a débuté le 2 Juillet 2018, et le piquetage a été fait à l'aide d'une ficelle, d'un décamètre et des piquets de bois. Tout au long du mètre linéaire, les graines du souchet ont été disposés l'une après l'autre dans les lits de semis pour les écotypes a et $\mathrm{c}$, alors que 2 à 3 graines de souchet pour les écotypes $b$ et $d$. Les mêmes quantités de graines par accession de souchet ont été pesées et semées sur chaque unité expérimentale à l'aide d'une balance. Le semis a eu lieu le 05 Juillet 2018 en une journée. La fertilisation s'est faite à la volée à base d'un engrais minéral dont la formulation était de NPKSB (22-10-15-5-1) à la dose de 2500 $\mathrm{kg} / \mathrm{ha}$. Le désherbage manuel a eu lieu le 13 Août 2018 à la main. Et la récolte a eu lieu le 13 Septembre 2018.

\section{Collecte des données}

Avant la date de la maturation de tubérisation des plants identifiables, les paramètres tels que la hauteur des plants, la longueur et la largeur des feuilles et le nombre de feuilles ont été mesurés à trois dates $(28$ Jours Après Semis (JAS) (Photo 3e), 42 JAS (Photo 3f) et 56 JAS) différentes pour la caractérisation des accessions. Les dates de la maturation des tubercules de chaque accession ont été évaluées sur chaque unité expérimentale afin d'apprécier le cycle de chaque écotype. Après la récolte, les tubercules de souchet récoltés sur chaque unité expérimentale ont été étalés et séchés durant 02 semaines au niveau du magasin avant d'être pesés. Les rendements en $\mathrm{kg}$ par unité expérimentale ont été évalués, les poids moyens de 100 grains choisis au hasard par unité expérimentale ont été également évalués. Tous les prix des intrants et des extrants ont été 
évalués au prix du marché au moment des opérations.

\section{Traitement et analyse des données}

Avant d'être analysés, tous les paramètres mesurés ont été ramenés aux mêmes unités. Les Analyses en Correspondances Multiples (ACM) ont été utilisées pour caractériser les quatre écotypes de souchet par rapport aux paramètres retenus. Avant d'évaluer la quantité nécessaire des inputs de la production des tubercules de souchet donnant les meilleures productivités marginales, l'ANOVA a été fait à l'aide du logiciel SPSS.20 pour évaluer les effets des accessions et des densités de semis sur les rendements moyens en t/ha des tubercules du souchet. Le logiciel GAMS a permis de ramener le problème sous forme de Programmation Linéaire (LP), afin de faciliter l'introduction des données. Les formulations mathématiques (équations) du problème suivantes ont été utilisées pour l'optimisation de tous les inputs disponibles afin d'estimer l'un des profits optimaux de la production des tubercules par accession.

\section{Fonction de l'objectif}

Cette fonction vise à maximiser le profit d'un producteur (bénéficiaire) par rapport au choix d'une accession, en tenant compte des ressources dont il dispose. Le profit est exprimé par la somme des revenus totaux moins la somme des coûts totaux induits dans la production des tubercules du souchet. Cette équation se matérialise par l'équation (1). La fonction de l'objectif à maximiser pour notre problème est :

Max

(1)

$$
\mathrm{Z}=\sum_{i=1}^{n} \operatorname{PiYiXi}-\sum_{i=1}^{n} \mathrm{Ci} \text {. }
$$

Avec $\mathrm{Pi}=$ prix de vente d'un sac de $100 \mathrm{~kg}$ des tubercules du souchet en FCFA de chaque producteur d'ordre $\mathrm{i}, \mathrm{Yi}=$ rendement moyen des tubercules du souchet en $\mathrm{Kg}$ de chaque producteur d'ordre $\mathrm{i}, \mathrm{X}_{\mathrm{i}}=$ superficie allouée à la production des tubercules du souchet en ha du même producteur d'ordre $\mathrm{i}$ et $\mathrm{Ci}=$ coûts de ses inputs en FCFA d'ordre i. Le Tableau 1 présente la synthèse de l'input/Output (Coût/bénéfice) de production de souchet. L'issu du Tableau 1 donne les différentes étapes de la mise en équation des variables retenues.

$>$ Définition des variables

$\mathrm{X}_{1}=$ quantité en $\mathrm{Kg}$ des tubercules de souchet produit par l'accession «a»

$\mathrm{X}_{2}=$ quantité en $\mathrm{Kg}$ des tubercules de souchet produit par l'accession «b»

$\mathrm{X}_{3}=$ quantité en $\mathrm{Kg}$ des tubercules de souchet produit par l'accession «c»

$\mathrm{X}_{4}=$ quantité en $\mathrm{Kg}$ des tubercules de souchet produit par l'accession «d»

$\mathrm{Z}=$ fonction de l'objectif de la maximisation de $\mathrm{Z}$

$>$ Mise en équation des variables définies :

Contraintes :

Disponibilité en travail (main d'œuvre):

$3174,68 \mathrm{X}_{1}+4309,6 \mathrm{X}_{2}+3956,89 \mathrm{X}_{3}+$

$3067,31 \mathrm{X}_{4} \leq 5000$

Disponibilité en capital pour l'achat des semences

$63212,5 \mathrm{X}_{1}+50400 \mathrm{X}_{2}+71456,67 \mathrm{X}_{3}+3237,5 \mathrm{X}_{4}$

$\leq 75000$

Disponibilité en capital pour la location de la parcelle

$4\left(\mathrm{X}_{1}+\mathrm{X}_{2}+\mathrm{X}_{3}+\mathrm{X}_{4}\right)$

(4)

Disponibilité en capital pour l'achat des éléments fertilisants (NPK et DAP):

$2\left(\mathrm{X}_{1}+\mathrm{X}_{2}+\mathrm{X}_{3}+\mathrm{X}_{4}+\mathrm{X}_{5}\right) \leq 2,1$

Disponibilité en capital pour l'amortissement des matériels et équipements

$7\left(\mathrm{X}_{1}+\mathrm{X}_{2}+\mathrm{X}_{3}+\mathrm{X}_{4}\right) \quad \leq 10$

(6)

Max Z $=135112,82 X_{1}+267330,4 X_{2}+503427,44$

$\mathrm{X}_{3}+511627,19 \mathrm{X}_{4}$

La non négativité des variables $\mathrm{X}_{1} ; \mathrm{X}_{2} ; \mathrm{X}_{3}$; $\mathrm{X}_{4} \geq 0$ 


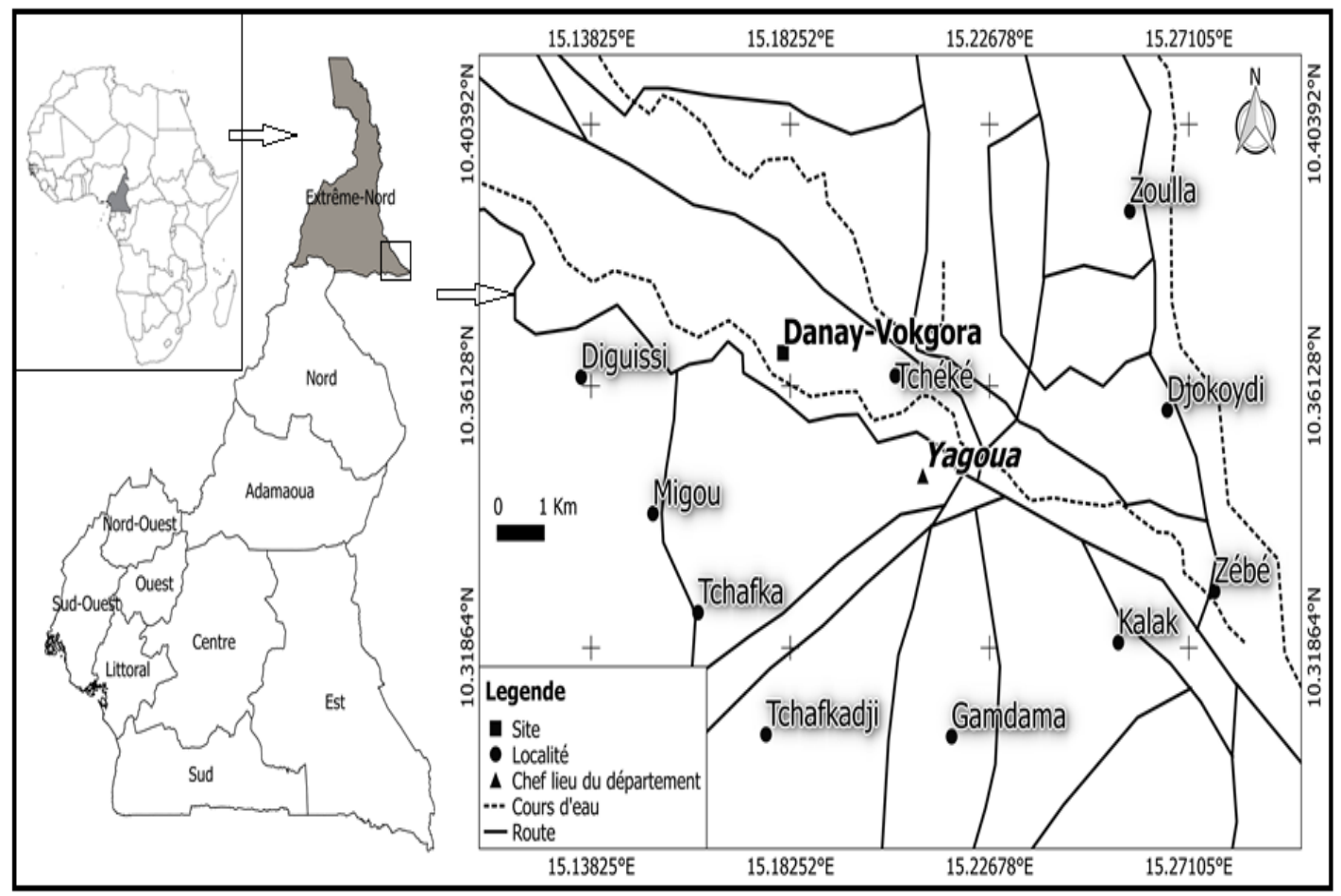

Figure 1 : Localisation du site d'étude (Source : Google Earth 2018 et données GPS).
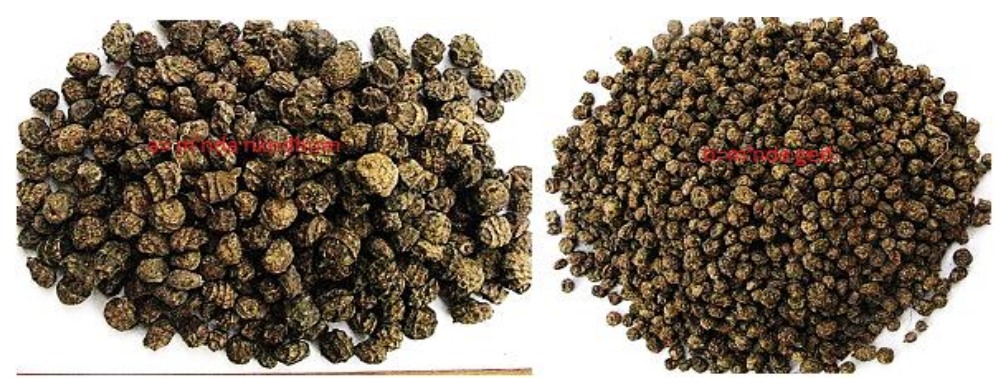

a) Gros grain

b) Petit grain
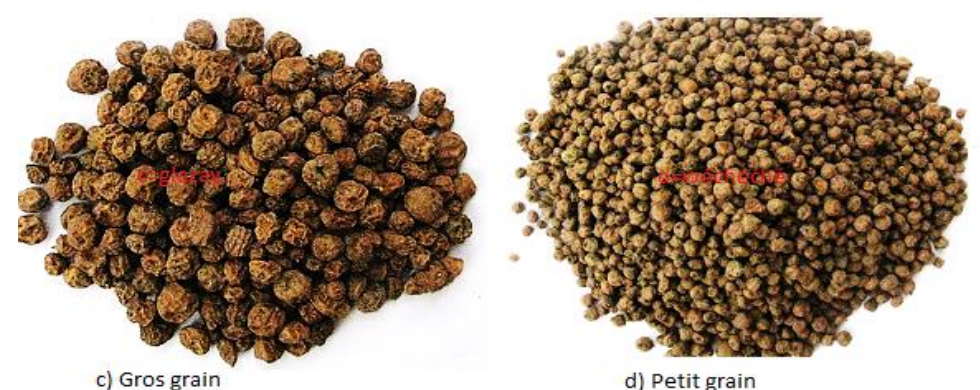

d) Petit grain

Photo 1 : différentes accessions collectées à l'état sec. 


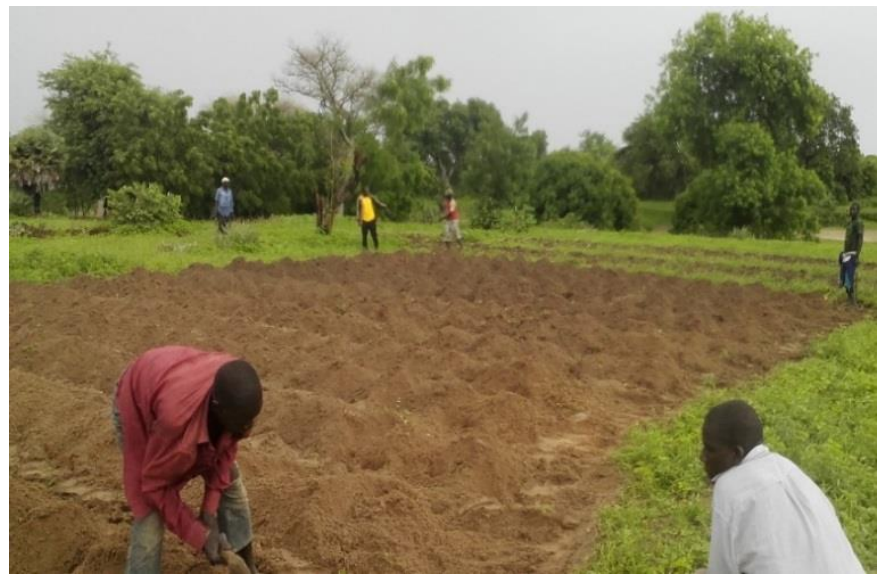

Photo 2 : Méthode de labour en monticule du champ expérimental.
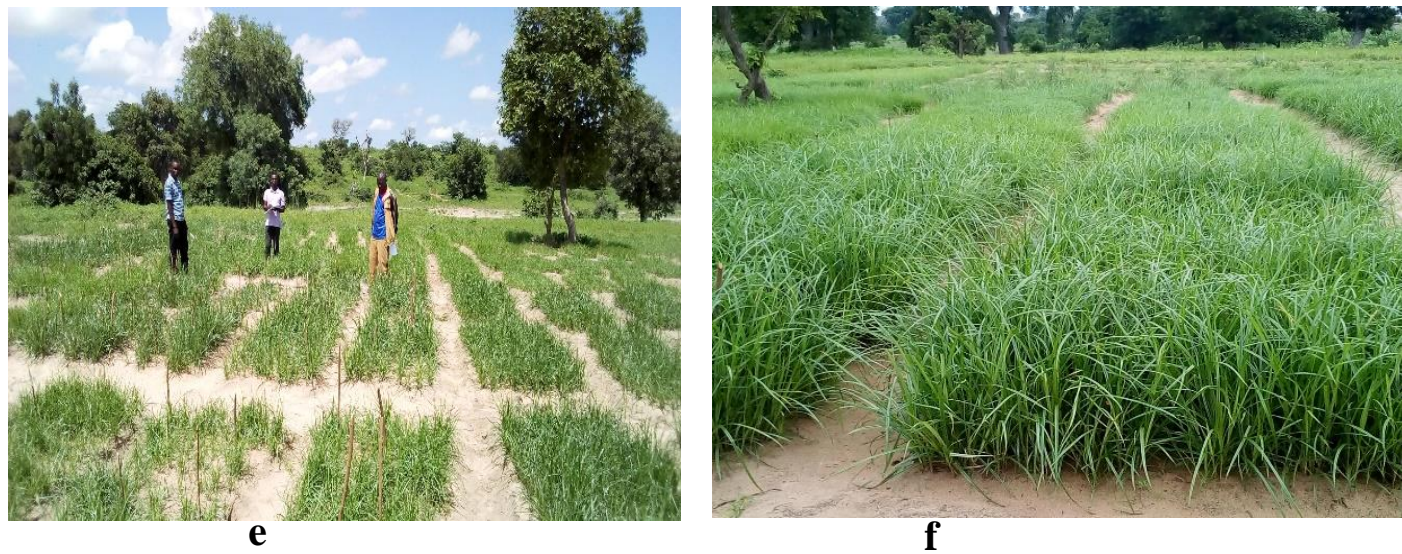

Photo 3 : Vues partielles du champ expérimental à 28 JAS et 42 JAS respectivement.

Tableau 1 : Synthèse des résultats de l'analyse coût-bénéfice par accession.

\begin{tabular}{|c|c|c|c|c|c|c|c|c|c|c|}
\hline $\begin{array}{l}\text { Accessions du } \\
\text { souchet }\end{array}$ & $\begin{array}{c}\text { M0 en } \\
\text { hj }\end{array}$ & $\begin{array}{l}\text { MO en } \\
\text { FCFA }\end{array}$ & $\begin{array}{l}\text { Coût des } \\
\text { semences } \\
\text { (FCFA) }\end{array}$ & $\begin{array}{c}\text { Eléments } \\
\text { fertilisants } \\
\text { (FCFA) }\end{array}$ & $\begin{array}{c}\text { Amortissements en } \\
\text { FCFA }\end{array}$ & $\begin{array}{c}\text { Location des } \\
\text { parcelles en } \\
\text { FCFA }\end{array}$ & $\begin{array}{l}\text { Rendements } \\
\text { en kg }\end{array}$ & $\begin{array}{l}\text { Prix du } \\
\text { kg en } \\
\text { FCFA }\end{array}$ & revenus & Profits \\
\hline a & 539,06 & 3174,68 & 63212,5 & 20000 & 7000 & 4000 & 232,5 & 1000 & 232500 & 135112,82 \\
\hline b & 731,77 & 4309,6 & 50400 & 20000 & 7000 & 4000 & 294,2 & 1200 & 353040 & 267330,4 \\
\hline c & 671,88 & 3956,89 & 71456,67 & 20000 & 7000 & 4000 & 415,8 & 1466,67 & 609841 & 503427,44 \\
\hline d & 520,83 & 3067,31 & 63237,5 & 20000 & 7000 & 4000 & 456,7 & 1333,33 & 608932 & 511627,19 \\
\hline Totaux & 850 & 5000 & 75000 & 21000 & 10000 & 5000 & 5000 & 1800 & & \\
\hline
\end{tabular}

NB : MO : Mains d'œuvre, hj : homme-jour, a=m'nda nkodhum ; b=m'nda ged ; c=glazay ; d=wécheché ; une superficie d'un $1 / 4$ ha coûte 5000 FCFA en Journée de Travail (JDT), les données totales du tableau 1 sont simulées à partir des coûts des opérations de la production du souchet de l'étude réalisée par Warouma et al.,(2013) au Niger dont leurs caractéristiques pédoclimatiques sont semblables à celui de l'Extrême-Nord. 


\section{RESULTATS}

\section{Caractérisation des accessions de souchet}

Le regroupement de 04 accessions de souchet collectées dans les différentes localités du Département du Mayo-Tsanaga se présente sur le diagramme de la normalisation principale de la variable (type d'écotype) (Figure 2).

L'issu de la Figure 2 a permis de regrouper la diversité de 04 accessions de souchet en 04 classes différentes selon l'axe de symétrie. L'accession $\mathrm{d}=$ Wéchéché constitue la classe 1, l'accession a= m'nda nkudhum constitue la deuxième classe 2 , l'accession $b=$ m'nda ged constitue la classe 3 et enfin la classe 4 correspond à l'accession $\mathrm{c}=$ glazay . Par ailleurs, les résultats montrent que l'axe 1 oppose les accessions de couleurs différentes (d, c et a, b respectivement noire claire et marron). Alors que l'axe de symétrie montre que les 04 accessions ( $a, b$, c et d) sont opposées diamétralement de part et d'autre par rapport à la grosseur des graines. Cette catégorisation des accessions de souchet se concentre sur certains paramètres agro morphologiques qui sont représentés sur la Figure 3 du diagramme de la normalisation des paramètres pris en compte.

Cette normalisation principale (Figure 3) montre que les paramètres mesurés sont regroupés en deux groupes, le premier groupe englobe Acc souchet, IFP42JAS, LGS, 1FP28JAS, LFP28JAS, DGS, TP28JAS, LFP42JAS, TP56JAS, NFP42JAS, et 1FP56JAS. Concernant le groupe 2, il est constitué de TP42JAS, DS, LFP56JAS et NFP56JAS. Les valeurs de matrice de corrélation (Tableau 2) entre ces paramètres montrent que ces deux groupes de caractères constitués présentent des corrélations fortes avec le rendement en $\mathrm{t} / \mathrm{ha}$ d'une part, et avec le poids en $\mathrm{kg}$ de 100 graines de souchet d'autre part. $\mathrm{Vu}$ certains paramètres, les largeurs des feuilles à 28, 42 et 56 Jours Après Semis (JAS) ont présenté des coefficients de corrélation fortes soient 0,$72 ; 0,68 ; 0,71 ; 0,57$ respectivement avec le rendement en t/ha. Alors qu'avec le poids en $\mathrm{kg} 100$ graines de souchet, les paramètres tels: Acc souchet,
TP28JAS, TP56JAS, LGS, DGS, IFP28JAS, 1FP42JAS, 1FP56JAS, LFP28JAS et LFP42JAS montrent des corrélations très élevées soient 0,$89 ; 0,57 ; 0,54 ; 0,88 ; 0,91$; 0,$88 ; 0,87 ; 0,56 ; 0,67 ; 0,55$ respectivement. Cette différenciation qui existe entre les accessions de souchet se traduit également par la plus petite variabilité des rendements en $\mathrm{t} / \mathrm{ha}$ à des différentes densités de semis. L'analyse de la variance a permis de tester l'hypothèse selon laquelle qu'il n'y'a pas des différences significatives entre les accessions testées du point de vue du rendement moyen en $t / h a$ et du poids moyen de 100 grains des tubercules des souchets à de différentes densités des semis. Les Tableaux 3 et 4 présentent les résultats de l'analyse de la variance (ANOVA, test de Fisher).

\section{Evaluation des rendements de souchet} Effet des traitements (densités et accessions) sur les rendements en t/ha de souchet

Les résultats du Tableau 3 montrent qu'il n'existe pas des différences significatives $\left(\ll \mathrm{a} »\left(\mathrm{p}_{1}=0,591\right) ; \ll \mathrm{b} »\left(\mathrm{p}_{2}=0,526\right) ; \ll \mathrm{c} »\left(\mathrm{p}_{3}=\right.\right.$ $\left.0,692) ; \ll d »\left(p_{4}=0,761\right)\right)$ en rendement moyen des tubercules de différentes accessions de souchet testées à des différentes densités de semis. Par ailleurs, des différences significatives $\left(\mathrm{D}_{1}(\mathrm{P}=0,017) ; \mathrm{D}_{2}(\mathrm{P}=0,02)\right.$ et $\mathrm{D}_{3}$ $(\mathrm{P}=0,032))$ en rendement moyen de souchet à différentes densités de semis $\left(D_{1}, D_{2}\right.$ et $\left.D 3\right)$ ont été enregistrées.

Les résultats du Tableau 4 montrent qu'au seuil de $5 \%$, le test de Fisher a révélé qu'il y'a des différences $(p=0,000)$ significative en poids de 100 grains des tubercules du souchet. Les accessions «a», «b» n'ont montré des différences entre elles, alors que les accessions «c» et «d» se sont différenciés entre elle et des deux autres du point de vue du poids de 100 graines des tubercules de souchet. Par ailleurs, les accessions «a» et «b» se sont différenciés des accessions $« \mathrm{c} »$ et $« \mathrm{~d} »$ du point de vue de rendement moyen en t/ha des accessions de souchet. Les accessions «c» et «d» ont présenté les meilleurs résultats de rendement moyen en t/ha et en poids de 100 graines des tubercules de souchet. Pour une bonne amélioration de ces 
rendements moyens en t/ha de souchet, la détermination de la meilleure combinaison des inputs de production permettrait de déterminer la valeur ajoutée sur le rendement.

\section{Evaluation de la meilleure combinaison des inputs de production de souchet}

La détermination de la meilleure combinaison des inputs de production du souchet indiqué dans le Tableau 5 montrent qu'il faut 2190,936 FCFA comme coût relative à la main d'œuvre, 45169,643 FCFA en faveur du capital pour l'achat des semences, 5000 FCFA pour le capital de la location de la parcelle, 14285,714 FCFA pour capital d'achat des éléments fertilisants et enfin 2857,143 FCFA pour la dotation aux amortissements des matériels et équipements. La simulation a montré que seule la productivité marginale «Shadow price» relative au capital de la location de la parcelle a présenté une valeur ajoutée de 73,090 FCFA. Ceci veut dire qu'une unité additionnelle relative à cette ressource procurerait 73,090 FCFA au profit optimal donc la valeur optimale du profit passera de 365 447,9929 FCFA à 365 521,083 FCFA pour la ressource utilisée. Quant aux autres ressources, leurs productivités marginales sont nulles. Ce qui veut dire que les ressources utilisés pour la production de chaque accession de souchet sont en excès. Alors, une unité supplémentaire de ces ressources n'ajouterait rien au profit optimal. La meilleure combinaison nécessaire des inputs a permis d'identifier la meilleure accession qui présente une valeur de production optimale du point de vue du rendement moyen.

L'issu des résultats du Tableau 6 des profits par accession de souchet montre qu'une des solutions optimales peut être déduite quand les coûts réduits (productivités marginales de produits) sont de l'ordre de 376500 FCFA, 244300 FCFA et $8 \quad 199,750 \quad$ FCFA respectivement pour les accessions «a»; «b»; et «c». Alors que l'accession «d» a un coût réduit nul (0) avec 0,714 comme quantité nécessaire. La comparaison des quantités nécessaires et des productivités marginales de la production des accessions de souchet ont montré qu'il serait donc plus profitable de produire avec l'accession «d». Cependant, si l'on force de produire avec les accessions «a»; «b» et «c», le profit optimal (365447,9929 FCFA) serait diminué respectivement de 376500 FCFA, 244300 FCFA et 8 199,750 FCFA d'où l'un des profits optimaux passera de l'ordre de -11 052,0071 FCFA, 121 147,9929 FCFA et 357 248,2429 FCFA respectivement. Bien que l'accession «d» étant jugé propice pour la localité, mais une bonne maitrise de la conduite des opérations culturales du souchet reste la clé pour mieux améliorer sa production.

\section{Niveau d'appropriation de la conduite des opérations culturales des souchets par les petits producteurs}

Sur un choix aléatoire simple de 20 personnes sur 34 identifiées lors de la conduite de cet essai, l'ensemble (20) de ces personnes ont été enquêtées pour apprécier leurs opinions par rapport à la production de la culture de souchet. L'issu du Tableau 7 montre qu'il y'a un pourcentage élevé des personnes de sexe masculin (75\%) et ils sont pour la plupart mariés (70\%). Sur 20 bénéficiaires, un taux élevé (80\%) des agro-éleveurs a été enregistré, cela démontre davantage que les activités d'agriculture et d'élevage vont de pair. Ce dualisme de l'agriculture et de l'élevage permet de diversifier des activités chez les petits producteurs, ce qui leur rapporte beaucoup de devises ou des revenus non négligeables par rapport aux autres activités rurales. Leur appréciation par rapport aux goûts et préférences des bénéficiaires ont montré que $70 \%$ des producteurs apprécient l'accession «c» grâce à sa sucrerie et sa valeur marchande. La technique de labour de la production ont montré qu'il y'a $85 \%$ des bénéficiaires qui préfèrent la méthode d'aménagement à la traction animale, comparativement à la méthode de labour en monticule. En plus, 70\% des bénéficiaires ont donné une réponse affirmative par rapport à leur capacité de conduire cette pratique culturale. 


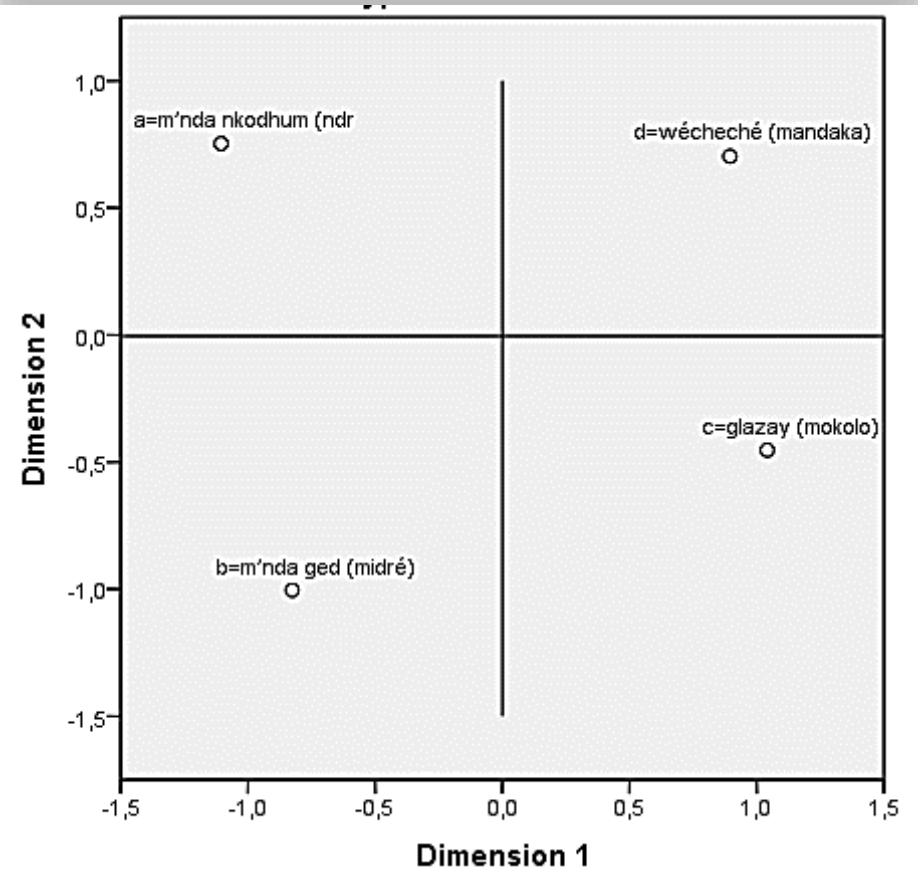

Figure 2 : Diagramme de la normalisation de 04 accessions de souchet.

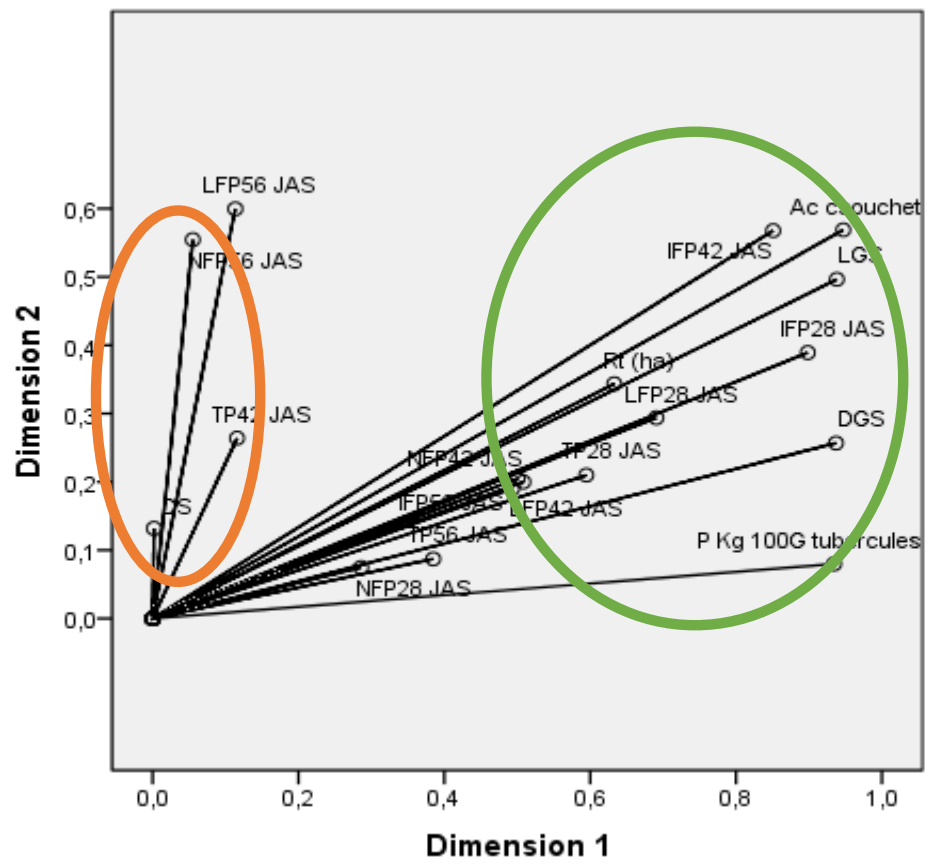

Figure 3 : Diagramme de la normalisation de quelques paramètres de maturation physique des souchets. 
Tableau 2 : matrice des corrélations des paramètres de croissance mesurés.

\begin{tabular}{|c|c|c|c|c|c|c|c|c|c|c|c|}
\hline & $\begin{array}{c}\text { type des } \\
\text { accessions } \\
\text { du souchet }\end{array}$ & $\begin{array}{c}\text { la taille des } \\
\text { plants à la } \\
\text { date du } 30 \\
\text { JAS }\end{array}$ & $\begin{array}{c}\text { la taille des } \\
\text { plants à la } \\
\text { date du } 47 \\
\text { JAS }\end{array}$ & $\begin{array}{l}\text { Longueur } \\
\text { graine de } \\
\text { semis } \\
\end{array}$ & $\begin{array}{l}\text { densité } \\
\text { graine } \\
\text { semis }\end{array}$ & $\begin{array}{c}\text { Nombre } \\
\text { des feuilles } \\
\text { des plants à } \\
\text { la date du } \\
\text { 30 JAS } \\
\end{array}$ & $\begin{array}{c}\text { Nombre } \\
\text { des feuilles } \\
\text { des plants } \\
\text { à la date } \\
\text { du } 47 \text { JAS } \\
\end{array}$ & $\begin{array}{c}\text { largeur des } \\
\text { feuilles des } \\
\text { plants à la } \\
\text { date du } 30 \\
\text { JAS } \\
\end{array}$ & $\begin{array}{c}\text { largeur des } \\
\text { feuilles des } \\
\text { plants à la } \\
\text { date du } 47 \\
\text { JAS } \\
\end{array}$ & $\begin{array}{c}\text { Longueur } \\
\text { des feuilles } \\
\text { des plants à } \\
\text { la date du } 30 \\
\text { JAS } \\
\end{array}$ & $\begin{array}{c}\text { longueur } \\
\text { des feuilles } \\
\text { des plants } \\
\text { à la date } \\
\text { du } 47 \mathrm{JAS} \\
\end{array}$ \\
\hline type des accessions du & & & & & & & & & & & \\
\hline souchet & 1 & & & & & & & & & & \\
\hline $\begin{array}{l}\text { la taille des plants à la date } \\
\text { du } 30 \text { JAS }\end{array}$ & 0,435 & 1 & & & & & & & & & \\
\hline $\begin{array}{l}\text { la taille des plants à la date } \\
\text { du } 47 \text { JAS }\end{array}$ & 0,258 & 0,438 & 1 & & & & & & & & \\
\hline Longueur graine de semis & 0,897 & 0,45 & 0,264 & 1 & & & & & & & \\
\hline densité graine semis & 0,887 & 0,505 & 0,222 & 0,892 & 1 & & & & & & \\
\hline $\begin{array}{l}\text { Nombre des feuilles des } \\
\text { plants à la date du } 30 \text { JAS }\end{array}$ & 0,288 & 0,303 & 0,19 & 0,145 & 0,171 & 1 & & & & & \\
\hline $\begin{array}{l}\text { Nombre des feuilles des } \\
\text { plants à la date du } 47 \text { JAS } \\
\text { largeur des feuilles des }\end{array}$ & 0,354 & 0,406 & 0,212 & 0,344 & 0,438 & 0,342 & 1 & & & & \\
\hline $\begin{array}{l}\text { plants à la date du } 30 \text { JAS } \\
\text { largeur des feuilles des }\end{array}$ & 0,884 & 0,567 & 0,261 & 0,784 & 0,864 & 0,374 & 0,513 & 1 & & & \\
\hline $\begin{array}{l}\text { plants à la date du } 47 \text { JAS } \\
\text { Longueur des feuilles des }\end{array}$ & 0,83 & 0,519 & 0,225 & 0,794 & 0,824 & 0,211 & 0,427 & 0,859 & 1 & & \\
\hline $\begin{array}{l}\text { plants à la date du } 30 \text { JAS } \\
\text { longueur des feuilles des }\end{array}$ & 0,637 & 0,738 & 0,395 & 0,57 & 0,673 & 0,348 & 0,419 & 0,782 & 0,728 & 1 & \\
\hline plants à la date du $47 \mathrm{JAS}$ & 0,545 & 0,647 & 0,677 & 0,508 & 0,562 & 0,333 & 0,406 & 0,635 & 0,557 & 0,652 & 1 \\
\hline
\end{tabular}

NB : JAS : Jours Après Semis 
Tableau 3 : Effet des accessions et des densités sur le rendement en t/ha des tubercules de souchet.

\begin{tabular}{lcccc}
\hline $\begin{array}{l}\text { Accessions/ } \\
\text { Densités de semis }\end{array}$ & $\begin{array}{c}\mathbf{a}=\mathbf{m}^{\prime} \text { nda nkodhum } \\
(\text { ndroukolay) (midré) }\end{array}$ & $\begin{array}{c}\mathbf{b}=\mathbf{m}^{\prime} \text { nda ged } \\
(\text { midré})\end{array}$ & $\begin{array}{c}\mathbf{c = g l a z a y} \\
(\text { mokolo) }\end{array}$ & $\begin{array}{c}\mathbf{d = w e ́ c h e c h e ́ ~} \\
(\text { mandaka) }\end{array}$ \\
\hline Témoin $(\mathrm{T})$ & $0,26 \pm 0,09^{\mathrm{aA}}$ & $0,27 \pm 0,02^{\mathrm{aA}}$ & $0,40 \pm 0,12^{\mathrm{aA}}$ & $0,59 \pm 0,04^{\mathrm{aA}}$ \\
Densité $\left(\mathrm{D}_{1}\right)$ & $0,23 \pm 0,04^{\mathrm{aA}}$ & $0,32 \pm 0,06^{\mathrm{abA}}$ & $0,38 \pm 0,06^{\mathrm{bA}}$ & $0,43 \pm 0,08^{\mathrm{cA}}$ \\
Densité $\left(\mathrm{D}_{2}\right)$ & $0,20 \pm 0,02^{\mathrm{aA}}$ & $0,29 \pm 0,02^{\mathrm{aA}}$ & $0,41 \pm 0,05^{\mathrm{bA}}$ & $0,46 \pm 0,10^{\mathrm{bA}}$ \\
Densité $\left(\mathrm{D}_{3}\right)$ & $0,23 \pm 0,05^{\mathrm{aA}}$ & $0,30 \pm 0,05^{\mathrm{abA}}$ & $0,46 \pm 0,09^{\mathrm{bA}}$ & $0,46 \pm 0,14^{\mathrm{bA}}$ \\
\hline
\end{tabular}

NB : Les lettres minuscules comparent les productions des différentes accessions à une densité fixe. Les lettres majuscules comparent les rendements moyens en t/ha des différentes densités pour une accession de souchet donnée. Les productions qui sont statistiquement homogènes au seuil de $5 \%$ de signification portent les mêmes lettres.

Tableau 4 : Effet des accessions sur le rendement en t/ha et sur le poids en g de 100 grains des tubercules de souchet.

\begin{tabular}{|c|c|c|c|c|}
\hline $\begin{array}{l}\text { Accessions } \\
\text { Paramètres mesurés }\end{array}$ & $\begin{array}{l}\mathbf{a}=\mathbf{m} \text { 'nda nkodhum } \\
\text { (ndroukolay) (midré) }\end{array}$ & 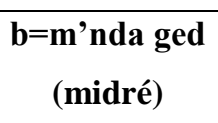 & $\begin{array}{l}\text { c=glazay } \\
\text { (mokolo) }\end{array}$ & $\begin{array}{c}\text { d=wécheché } \\
\text { (mandaka) }\end{array}$ \\
\hline Rendement en $\mathrm{t} / \mathrm{ha}$ & $0,23 \pm 0,05^{\mathrm{a}}$ & $0,29 \pm 0,04^{\mathrm{a}}$ & $0,42 \pm 0,08^{b}$ & $0,46 \pm 0,18^{b}$ \\
\hline Poids de 100 grains (g) & $11,34 \pm 1,16^{\mathrm{a}}$ & $10,58 \pm 0,95^{\mathrm{a}}$ & $65,68 \pm 5,43^{b}$ & $70,60 \pm 4,20^{\mathrm{c}}$ \\
\hline
\end{tabular}

NB : Les lettres comparent les rendements moyens (t/ha) des accessions du souchet. Les rendements qui sont statistiquement homogènes au seuil de $5 \%$ de signification portent la même lettre.

Tableau 5 : Différents niveaux des ressources de la production des tubercules de souchet selon le résultat du modèle de GAMS.

\begin{tabular}{lccc}
\hline Ressources & $\begin{array}{c}\text { Quantités } \\
\text { disponibles }\end{array}$ & $\begin{array}{c}\text { Quantités } \\
\text { nécessaires }\end{array}$ & $\begin{array}{c}\text { Productivités } \\
\text { marginales }\end{array}$ \\
\hline Mains d'œuvre (Homme jour) & 5000 & 2190,936 & 0 \\
Capital pour l'achat des semences (FCFA) & 75000 & 45169,643 & 0 \\
Capital pour la location de la parcelle (FCFA) & 5000 & 5000 & 73,090 \\
Capital pour les éléments fertilisants (FCFA) & 21000 & 14285,714 & 0 \\
Amortissements des matériels et équipements (FCFA) & 10000 & 2857,143 & 0 \\
\hline
\end{tabular}

Tableau 6 : Différents niveaux de production des accessions de souchet en fonction des quantités (disponible et utilisé) et productivités marginales du modèle du GAMS.

\begin{tabular}{lccc}
\hline $\begin{array}{l}\text { Quantites (fcfa) produites } \\
\text { par accession }\end{array}$ & $\begin{array}{c}\text { Quantités } \\
\text { disponibles }\end{array}$ & $\begin{array}{c}\text { Quantités } \\
\text { nécessaires }\end{array}$ & $\begin{array}{c}\text { Productivités } \\
\text { marginales }\end{array}$ \\
\hline $\mathrm{a}=$ m'nda nkodhum & 0 & 0 & -376500 \\
$\mathrm{~b}=$ m'nda ged & 0 & 0 & -244300 \\
c=glazay & 0 & 0 & $-8199,750$ \\
$\mathrm{~d}=$ wécheché & 0 & 0,714 & 0 \\
\hline
\end{tabular}


Tableau 7 : Profils des bénéficiaires de la pratique culturale de la culture de souchet.

\begin{tabular}{llll}
\hline Parametres mésurés & \multicolumn{1}{c}{ Modalités } & Effectifs & Pourcentages (\%) \\
\hline Sexes & Masculin & 15 & 75 \\
& Feminin & 5 & 25 \\
\hline Religions & Chretien & 15 & 75 \\
& Musulman & 4 & 20 \\
& Animisme & 1 & 5 \\
\hline Statuts matrimoniaux & Celibataire & 6 & 30 \\
& Marié & 14 & 70 \\
& Divorcé & 0 & 0 \\
& Veuf(ve) & 0 & 0 \\
\hline Niveaux d'education & Primaire & 10 & 50 \\
& Secondaire & 8 & 40 \\
& illettré & 2 & 10 \\
\hline Appreciation des accessions & a=m'nda nkodhum & 2 & 10 \\
& b=m'nda ged & 0 & 0 \\
& c=glazay & 14 & 70 \\
& d=wécheché & 4 & 20 \\
\hline Mode d'amenagement de la parcelle & attraction animale & 17 & 85 \\
& labour en monticule & 3 & 15 \\
& (manuelle) & & \\
\hline Capacité de conduite de l'itineraire & Oui & 14 & 70 \\
technique du souchet & Non & 6 & 30 \\
\hline Activités principales & Agro-éléveur & 16 & 80 \\
& Elevage & 4 & 20 \\
\hline
\end{tabular}

\section{DISCUSSION}

Caractérisation des accessions de souchet.

L'analyse de la caractérisation a permis de regrouper les accessions de souchet en 04 groupes qui sont constitués de la classe 1, 2, 3 et 4 respectivement pour les accessions «d»= Wéchéché, «a»= m’nda nkudhum, «b»= m'nda ged et «c»= glazay. Les 04 classes des accessions de souchet testées se sont différenciées du point de vue des paramètres agro morphologiques pris en compte. Cette division des accessions de souchet s'est faite selon l'axe de symétrique du diagramme de la normalisation d'une part. D'autre part, l'axe 1 oppose les accessions de souchet des couleurs différentes (noire claire $(« a », \ll b »)$ et marron $(« \mathrm{~d} »,\langle\mathrm{c} »))$. Par contre au Niger, l'analyse de la typologie locale de la culture des souchets de Bori et al. (2018) ont montré qu'elle est composée de 02 catégories (gros tubercules et petits tubercules). Des études similaires de Warouma et al. (2015), Laouali et al. (2011) cité par Bori et al. (2018) et Christian et al. (2013) ont également classées les accessions en deux groupes, et ont montré que l'existence de ces deux types de souchet était qualifiée de «sous espèces». Par ailleurs, les paramètres agro morphologiques mesurés au niveau des différentes accessions de souchet sont regroupés en deux (02) grands groupes. Ces groupes 1 et 2 ont enregistré 11 et 04 paramètres respectivement. Ces deux groupes de paramètres agro morphologiques des accessions de souchet constituées ne respectent pas la classification selon la performance de croissance et du rendement. Ce qui implique une diversification des paramètres de croissance et du rendement ( $\mathrm{t} / \mathrm{ha}$ ) par rapport à la normalisation des caractères étudiés. Par conséquent, une diversification agro 
morphologique des accessions de souchet a été enregistrée du point de vue des paramètres mesurés. Les études d'évaluation de quinze génotypes de niébé de Lalsaga et Drabo (2017) au Burkina-Faso, ont permis d'identifier deux groupes sur la base des caractères quantitatifs réalisé à l'aide de l'ACP. Au Bénin, une grande variabilité morphologique et agronomique a été observée entre les accessions de voandzou (Karikari, 2000). Mais, les études de diversité génétique réalisées à l'aide de marqueurs neutres (protéines de réserves, isozymes, RAPD et AFLP) n'ont pas présenté cette diversification entre les accessions du voandzou (Massawe et al., 2002, Ntundu et al., 2004). Cependant, des travaux menés sur le voandzou par Djè et al. (2005) et Ofori et al. (2006) ont montré l'existence d'une importante variabilité aussi bien au niveau morphologique que moléculaire. Par ailleurs en Côte d'Ivoire, Coulibaly et al. (2019) ont observé la variabilité au niveau des stades phénologiques chez 15 lignées de la tomate, dont cela pourrait être liée à l'interaction des différents génotypes et l'environnement. L'analyse de la diversité agro morphologique de 54 accessions des maniocs testés au Burkina-Faso ont montré que la descriptive des caractères qualitatifs a révélé un polymorphisme entre les accessions. Ils ont constaté que la diversité morphologique potentielle est comparable entre les groupes et qu'il y'a relativement peu de différenciation dans l'étendue de l'espace morphologique des accessions maniocs (Gmakouba et al.,2018). Certainement, sous l'effet des sélections massales des producteurs, le souchet présente des écotypes locaux qui ont accumulé une grande diversité génétique qui a été très peu étudiée scientifiquement et surtout mal exploitée dans le programme de la sélection variétale en zone soudano-sahélienne du Cameroun. Des valeurs élevées de coefficient de corrélation ont été enregistrées entre certains paramètres de croissance avec celui du rendement en $t / h a$. Ces fortes valeurs des coefficients expliquent les relations des causes à effet entre la biomasse sèche et le rendement à la récolte. Ces corrélations constituent un outil indispensable pour les améliorateurs dans le choix des caractères à intégrer dans les programmes de sélection. Plus la biomasse sèche est élevée, plus le rendement moyen en t/ha est élevé. Seule l'accession «d» a présenté un rendement moyen élevé $(456,7 \mathrm{~kg} / \mathrm{ha}) \mathrm{par}$ rapport aux accessions de souchet. Au Niger, Bori et al. (2018) ont montré dans la région de Maradi que le plus petit rendement moyen est de $1069 \mathrm{~kg} / \mathrm{ha}$ alors que le rendement le plus élevé observé est de $4254 \mathrm{~kg} / \mathrm{ha}$. Parallèlement dans la région de Dosso, le plus petit rendement moyen est de $230 \mathrm{~kg} / \mathrm{ha}$ contre $3515 \mathrm{~kg} / \mathrm{ha}$ correspondant à la plus grande valeur de rendement moyen. Cette variabilité de rendement qui existe entre ce deux pays subsahariens (Cameroun et Niger) pourrait s'attribuer d'autre part à la gestion rationnelle des inputs de production de souchet qui reste un facteur prioritaire.

\section{Evaluation des quantités nécessaires des inputs de production.}

L'évaluation de la performance économique des accessions de souchet selon le modèle de la Programmation Linéaire nous montre que l'accession «d» (Wéchéché) (Photo1d) serait plus adaptée à cette localité de Danay-vokgora avec une valeur ajoutée marginale (Shadow price) de 73,090 FCFA relative au capital de la location de la parcelle. Ce qui implique un coût réduit égal à zéro en faveur de cette accession de souchet. Or les bénéficiaires de l'innovation (itinéraire technique) ont porté leur choix (goûts et préférences des consommateurs) sur l'accession «c» glazay (photo1c). Bien que le rendement moyen en t/ha de celle-ci (accession «c» glazay) soit moins important par rapport à l'accession «d». Les valeurs des profits de l'analyse coût/bénéfice sur un hectare sont de l'ordre de 503 427,44 FCFA et 511 627,19 FCFA respectivement pour les accessions «C» et «d». Cette différence qui existe entre les profits de ces accessions est de 8199,75 FCFA; cette différenciation en valeur correspond exactement à la valeur du coût réduit de l'accession «c». Ce manque à gagner de l'accession «c» par rapport à l'accession «d» constitue le coût d'opportunité par rapport à l'accession «d». Par conséquent, les 
producteurs de Danay-vokgora auront à choisir entre les accessions $« \mathrm{c} »$ et «d» en considérant cette valeur ajoutée par rapport à leur choix de l'accession «c». Par ailleurs, les études récentes de Bori et al. (2018) au Niger ont montré que le revenu moyen d'un producteur de la culture de souchet à gros tubercule était estimé à $452461 \mathrm{FCFA} / \mathrm{ha}$. Cette valeur de revenu du producteur du Niger est légèrement inférieure à celui obtenu au Cameroun, ceci serait dû à la quantité élevée des tubercules offerts sur les marchés nigériens par rapport à celui du Cameroun. Par conséquent, les tubercules sont plus chers (flambée du prix) au Cameroun qu'au Niger. En outre, au Niger les études de Warouma et al. (2015) ont montré que le prix de revient d'un sac de gros tubercules de souchet sur les marchés varie entre 25000 et 30000 F CFA. Ce qui met en évidence que la chaîne de valeur de l'exploitation de la culture de souchet est rentable à chaque niveau du maillon de la chaîne. Cela correspond à la réponse de l'une des femmes grossistes de la vente de souchet (Cyperus esculentus L.), qui a montré l'importance économique de cette culture négligée et sous utilisée, en déclarant: «Je ne laisserai jamais la vente de souchet par rapport à d'autres choses, parce qu'au cours de sa période de récolte, les vendeurs de pagne par exemple ne peuvent me rivaliser en termes $d u$ gain $(13100 \mathrm{f}$ /jour et $52400 \mathrm{f}$ tous les samedis pour la tontine). Quand je vends aux grossistes du Gabon et du Cameroun, je peux facilement réaliser un bénéfice de 262000 f/semaine» cette déclaration montre clairement l'analyse financière d'un exploitant de souchet (Dansi et al., 2012). De plus, les chercheurs à travers les études de marché ont montré également que la production de souchet constitue une source importante de revenus des ménages et contribue sensiblement à la réduction de la pauvreté. Certaines revues de la littérature disponibles ont démontrés également que ces espèces sont pour la plupart des espèces riches en nutriments et possèdent certaines vertus médicinales, leur utilisation permettant ainsi de lutter contre la malnutrition et d'améliorer l'état de santé des populations locales. Pareillement aux cultures négligées et sous utilisées, des résultats des analyses coût/bénéfice d'un producteur de voandzou au Bénin ont montré un profit net qui varie entre 259550 FCFA et 284500 FCFA par hectare (Gbaguidi et al., 2016). Cette valeur de la profitabilité de voandzou est faible par rapport au gain obtenu de la culture de souchet au Cameroun et au Niger, ceci est dû au déficit de la production et à la demande accrue en consommation de cette culture souchet par rapport à celle de voandzou. Il a été démontré également que la commercialisation du voandzou est génératrice de revenu des petits producteurs par ce qu'un sac de $100 \mathrm{~kg}$ de voandzou vendu génèrerait une hausse de la valeur ajoutée variant entre 2000 FCFA à 4000 FCFA des bénéfices en faveur des agriculteurs.

\section{Conclusion}

Dans le but de diversifier les cultures dans le département du Mayo-Danay, une étude portant sur la valorisation de certaines accessions de souchet a été réalisée en les introduisant dans la localité de DanayVokgora. Au terme de cette étude, 90\% des bénéficiaires ont capitalisés les connaissances sur la conduite de la culture de souchet. Les accessions de souchet ont montré une diversification génétique au sein des accessions testées du point de vu des paramètres de croissance et de rendement. Les accessions «c» (Glazay) et «d» (Wéchéché) ont présenté des meilleurs rendements en t/ha, mais seule l'accession «d» (Wéchéché) a présenté une productivité marginale «shadow price» donnant une meilleure solution optimale avec la bonne combinaison des inputs de production. Par conséquent, il serait mieux d'introduire et de produire avec l'écotype «d» malgré que $70 \%$ des bénéficiaires ont porté leurs choix sur l'accession «c». Les caractères d'intérêt analysés peuvent ainsi constituer des critères de base pour différencier les variétés et peuvent être exploités dans les programmes d'amélioration variétale de souchet au Cameroun. Pour parfaire cette étude, il serait judicieux de répliquer dans plusieurs zones et surtout d'évaluer cette pratique culturale à la fin de la campagne prochaine pour mieux apprécier le degré d'adoption de l'innovation 
relative à l'itinéraire technique de la culture de souchet.

\section{CONFLIT D'INTERETS}

Les auteurs déclarent qu'ils n'ont pas d'intérêts contradictoires.

\section{CONTRIBUTIONS DES AUTEURS}

DPS a collecté, analysé les données et a rédigé le papier. $\mathrm{AAB}, \mathrm{JPOL}$ et JB ont participé à la correction et l'amendement du manuscrit, OP et SB ont cherché le financement, participé à la collecte des données et au suivi technique de l'essai.

\section{REMERCIEMENTS}

Nos remerciements vont particulièrement à l'endroit de Monsieur GARANGSOU Clement et des populations de Danay-Vokgora pour avoir mobiliser des fonds, leur dynamisme et l'octroi de la parcelle pour la conduite de cet essai.

\section{RÉFÉRENCES}

De Castro O, Roberta Ga, Del Guacchio E, Paolo C, Paolo DL. 2015. A molecular survey concerning the origin of Cyperus esculentus (Cyperaceae, Poales), two sides of the same coin (weed vs. crop). Annals of Botany, 115: 733-745. DOI: $10.1093 / \mathrm{aob} / \mathrm{mcv} 001$

Aoua D, Dramane K, Fatoumata K, Mady S. 2008. Appui au développement de la chaîne de valeur du pois sucré à Farakala, Sikasso-Mali. 12p. DOI: https://docplayer.fr/48996269

Defelice. 2002. Yellow nutsedge (Cyprus esculentus L). Snack food of the gods. Weed Technology, 16: p901-907. DOI: https://doi.org/10.1614/0890037X(2002)016[0901:YNCELS]2.0.CO; 2

Warouma A, Lawali S, Saadou M. 2015. Investigations relatives à la conception d'un équipement amélioré pour le semis et la récolte du souchet (Cyperus esculentus L.) au Niger. Bulletin de la Recherche Agronomique du Benin, 74 : 17.

DOI : http://www.slire.net/download/2174/artic le_1_complet_brab_n_74_d_cembre_20

13_warouma_et_al.pdf

Bohen C, Wirth J. 2013. Souchet comestible (Cyperus esculentus L.) : Situation actuelle en suisse. Recherche Agronomique Suisse, 4 : 11-12. DOI : https://ira.agroscope.ch/enUS/publication/33027

Garba BA. 2007. Expérience de commercialisation de produits agricoles par le mécanisme du warrantage au sein de la fédération SA'A : Valorisation d'expériences d'organisations paysannes «Accès au marché et commercialisation de produits agricoles ». Forum régional Bamako.

http://www.ruralfinanceandinvestment.or g/sites/default/files/72011_Rapport.pdf

CSA. 2011. Sécurité alimentaire et Organisations Intermédiaires : évaluation et identification des besoins de renforcement des capacités des organisations paysannes dans six pays de l'UEMOA de la CEDEAO. Rapport Niger. http://www.csabe.org/IMG/pdf_Rapport_Niger_FINAL. pdf

Bori H, Toudou O, Moumouni DMA, Adam T. 2018. La culture de souchet (Cyperus esculentus) au Niger : Origine, atouts et contraintes. Journal of Animal \& Plant Sciences, 37(1): 5997-6007. DOI: https://reca-

niger.org/IMG/pdf/souchet_au_niger_20 18.pdf

Dodet M. 2006. Diversité génétique et phénologie de Cyperus esculentus L. (Cypéracées) pour une gestion intégrée de l'espèce dans les cultures de haute lande. Ecology, environment. Université de Bourgogne. 226pp. https://tel.archivesouvertes.fr/tel-00261512/document

Mulligan GA, Junkins BE. 1976. The Biology of Canadian Weeds. 17. Cyperus esculentus L. Canadian Journal of Plant Science, 56(2): 339-350. DOI: $10.4141 /$ cjps $76-052$

Ong peamuru. 2013. le souchet: (Cyperus esculentus) une culture de rente très prometteuse dans la région de Maradi (Niger). Rapports d'enquête sur le 
souchet. $\quad 2 \mathrm{p} . \quad$ https://recaniger.org/IMG/pdf/Souchet_au_Niger_P EAMURU_light.pdf

SNV. 2006. Rapport de l'atelier de mise en place de l'organisation sous régional des acteurs de la filière souchet en Afrique de l'ouest) Maradi (réalisé avec la chambre régional d'agriculture de Maradi). documents.worldbank.org>

502160REVISED00Rapport0060090fren ch

Baobab de saveurs. 2012. Fiche technique de l'huile de souchet. http://baobab-dessaveurs.com/wp-

content/uploads/2015/08/Fichetechnique-de-lhuile-de-souchet.pdf

Dansi R, Vodouhe P, Azokpota H, Yedomonhan P, Assogba A, Adjatin YL, Loko I, Dossou-Aminon, Akpagana K. 2012. Diversity of the Neglected and Underutilized Crop Species of Importance in Benin. The Scientific World Journal, 2012: 19p. DOI:10.1100/2012/932947.

Karikari KS. 2000. Variability between local and exotic Bambara groundnut landraces in Botswana. African Crop Science Journal, 8(2): 145-152. DOI : http://dx.doi.org/10.4314/acsj.v8i2.2770 4

Djè Y, Bonny BS, Zoro BIA. 2005. Observations préliminaires de la variabilité entre quelques morphotypes de voandzou (Vigna subterranea L. Verdc., Fabaceae) de Côte d'Ivoire. Biotechnol. Agron. Soc. Environ., 9(4) : 249-258.

DOI : https://www.researchgate.net/publication /26415371

Gbaguidi IA, Dossou-Aminon AP, Agre A, Dansi P, Rudebjer RH, Vodouhe R. 2016. Promotion de la chaine des valeurs des espèces négligées et sous utilisées au Bénin : cas du voandzou (Vigna subterranea L. Verdc.). International Journal of Neglected and Underutilized Species, 2: 19-32. DOI: https://docplayer.fr/53500677
Massawe FJ, Collinson ST, Roberts JA, Azam ASN. 1999. Effect of presowing hydration on germination, emergence and early growth of bambara groundnut (Vigna subterranea L. Verdc.). Seed Sci. Technol., 27: 893-905. DOI: https://books.google.cm/books?id=33qJF qmTZJQC\&pg=PA20\&lpg=PA20\&dq

Ntundu WH, Shillah SA, Marandu WYF, Christiansen JL. 2006. Morphological diversity of Bambara groundnut [Vigna subterranea (L.) Verdc.] Landraces in Tanzania. Genetic Resources and Crop Evolution, 53: 367-378. DOI: 10.1007/s10722-004-0580-2

Ofori K, Kumaga FK, Tonyigah A. 2006. Morphological characterization and agronomic evaluation of bambara groundnut (Vigna subterranea (L). Verd.) germplasm in Ghana. Plant Genetic Resources Newsletter, 145: 23-28. http://agris.fao.org

Gmakouba T, Koussao S, Traore ER, Kpemoua KE, Zongo JD. 2018. Analyse de la diversité agromorphologique d'une collection de manioc (Manihot esculenta Crantz) du Burkina Faso. Int. J. Biol. Chem. Sci., 12(1): 402-421. DOI: https://dx.doi.org/10.4314/ijbcs.v12i1.32

Oloukoi J, Yabi I, Houssou CS. 2019. Perceptions et stratégies paysannes d'adaptation à la variabilité pluviométrique au Centre du Bénin. Int. J. Biol. Chem. Sci., 13(3): 1366-1387. DOI: https://dx.doi.org/10.4314/ijbcs.v13i3.13

Coulibaly ND, Fondio L, FDP N'gbesso M, Doumbia B. 2019. Evaluation des performances agronomiques de quinze nouvelles lignées de tomate en station au centre de la Côte d'Ivoire. Int. J. Biol. Chem. Sci., 13(3): 1565-1581. DOI: https://dx.doi.org/10.4314/ijbcs.v13i3.29

Lalsaga WJA, Drabo I. 2017. Évaluation de quinze génotypes de niébé [Vigna unguiculata (L.) Walp.] Sous conditions pluviales dans le Nord et le Centre Ouest du Burkina Faso. Int. J. Biol. Chem. Sci., 11(6): 2756-2763. DOI: https://dx.doi.org/10.4314/ijbcs.v11i6.16 